\title{
miR-27a in serum acts as biomarker for prostate cancer detection and promotes cell proliferation by targeting Sprouty 2
}

\author{
WEIYIN GAO*, ZHENGDONG HONG*, HONGWEI HUANG, ANYI ZHU, SHUANGQUAN LIN, \\ CHENG CHENG, XIAO ZHANG, GAODE ZOU and ZIMIN SHI
}

Department of Urology Surgery, The Second Affiliated Hospital of Nanchang University, Nanchang, Jiangxi 330001, P.R. China

Received August 2, 2016; Accepted September 1, 2017

DOI: $10.3892 / 01.2018 .9274$

\begin{abstract}
Prostate cancer (PCa) exhibits a high incidence among men, but there is no effective and non-invasive biomarker for the diagnosis of $\mathrm{PCa}$, and the pathogenesis of PCa remains unclear. The present study identified that miR-27a was significantly overexpressed in the tumor tissues and sera of patients with PCa. In addition, high serum levels of miR-27a were correlated with poor survival in patients with PCa. Receiver-operating characteristic curves analysis demonstrated that the serum levels of miR-27a exhibited a high area under the curve value. Furthermore, miR-27a mimics or inhibitors significantly promoted or repressed the proliferation of PCa cells, respectively. In addition, it was identified that the expression of Sprouty2 (SPRY2) was inversely correlated with the expression of miR-27a in PCa tissues. The knockdown or overexpression of SPRY2 promoted or suppressed the proliferation of PCa cells, respectively, and the overexpression of SPRY2 inhibited the increased proliferation and cell cycle distribution of PCa cells mediated by miR-27a mimics. Taken together, these data indicated that the serum levels of miR-27a may be a novel and non-invasive biomarker for the diagnosis and prognosis of patients with PCa, and miR-27a/SPRY2 may be a therapeutic target for the treatment of $\mathrm{PCa}$.
\end{abstract}

\section{Introduction}

Prostate cancer (PCa) is the most commonly diagnosed malignancy among African American men and the second-leading cause of cancer-associated mortality during 2013 (1). For

Correspondence to: Dr Zimin Shi, Department of Urology Surgery, The Second Affiliated Hospital of Nanchang University, 1 Min De Road, East Lake District, Nanchang, Jiangxi 330001, P.R. China

E-mail: ncshizhimin@126.com

*Contributed equally

Key words: microRNA-27a, biomarker, prostate cancer, Sprouty2
African Americans, the overall 5-year survival rate of patients with prostate cancer is $100 \%$ when these tumors are diagnosed at early stages, yet when the cancer has spread to distant sites the 5-year survival rate decreases to $27 \%$ (1). Although the present biomarker for the diagnosis of $\mathrm{PCa}$ is prostate-specific antigen (PSA), it has been demonstrated that PSA is not associated with rates of mortality in PCa after 13 years (2). The negative consequences of PSA screening lead to over-diagnosis, overtreatment and treatment complications (3). Therefore, it is urgent to investigate novel biomarkers for the diagnosis of early PCa and explore novel therapeutic targets for the treatment of advanced PCa.

MicroRNAs (miRNAs/miRs), small noncoding single RNAs measuring 21-25 nucleotides in length, are stably expressed in clinical specimens, including serum (4), plasma (5) and urine (6). miRNAs serve essential regulatory roles through sequence-specific base pairing on the $3^{\prime}$ untranslated region of mRNAs, resulting in mRNA degradation or the inhibition of translation (7). Several studies have revealed that prostate cancer exhibits specific expression profiles of miRNAs, including miR-106a, miR-223, miR-20a, miR-21, miR-141 and miR-27a (8-10). Among these miRNAs, the downregulation of miR-27a was identified in high grade of prostate cancer (10), but Fletcher et al (11) demonstrated that androgen-regulated miR-27a acted as an oncogenic miR (oncomiR) and increased prostate cancer cell growth via targeting the tumor suppressor and androgen receptor corepressor, prohibitin. In other types of cancer, including pancreatic cancer (12), renal cell carcinoma (13) and osteosarcoma (14), miR-27a serves as an oncomiR and is involved in cell proliferation, colony formation and metastasis. However, in hepatocellular carcinoma, miR-27a was demonstrated to be downregulated and to suppress tumor metastasis by inhibiting epithelial-mesenchymal transition (15). Therefore, the present study focused on miR-27a, and aimed to investigate its expression and role in $\mathrm{PCa}$.

In the present study, it was identified that miR-27a was overexpressed in the tumor tissue and serum of patients with $\mathrm{PCa}$. The overexpression of miR-27a was associated with poor survival of patients and an increase tumor cell proliferation. Furthermore, it was identified that Sprouty2 (SPRY2) is a direct target of miR-27a, and the induced expression of SPRY2 
may rescue the miR-27a-mediated increase in tumor cell proliferation of PCa cells.

\section{Materials and methods}

Prostate carcinoma specimens and cell lines. All specimens were collected from the individuals who provided written informed consent according to the protocols approved by the Ethics Review Board at Nanchang University (Nanchang, China). A total of 60 patients (aged between 60 and 78, median 69 years) with PCa and 60 healthy subjects from the Second Affiliated Hospital of Nanchang University (Nanchang, China) were included in this study between March 2013 and June 2015. Three years of follow-up of the patients with PCa were performed. The serum samples were collected from PCa patients with different Tumor Node Metastasis (TNM) stages (16), stage I (12 patients), stage II (13 patients), stage III (25 patients), stage IV (10 patients). No patients underwent any treatment prior to the collection of serum samples. There was no significant difference in the age distribution between the patients with PCa and healthy subjects (data not shown). Cell-free serum was isolated from $5 \mathrm{ml}$ blood of patients and healthy subjects within $2 \mathrm{~h}$ via a two-step protocol $(1,500 \mathrm{xg}$ for $10 \mathrm{~min}$, followed by $12,000 \mathrm{x} \mathrm{g}$ for $2 \mathrm{~min}$, at $4^{\circ} \mathrm{C}$ ) (17). Finally, $450 \mu 1$ serum was moved into nuclear-free tubes and stored at $-80^{\circ} \mathrm{C}$.

Human PCa LNCaP and PC-3, and normal prostate epithelial RWPE-1 cell lines were purchased from American Type Culture Collection (Manassas, VA, USA). The cells were cultured in RPMI-1640 medium (Hyclone; GE Healthcare Life Sciences, Logan, UT, USA) supplemented with $15 \%$ fetal bovine serum (Hyclone; GE Healthcare Life Sciences) at $37^{\circ} \mathrm{C}$ in $5 \% \mathrm{CO}_{2}$.

RNA isolation. Circulating RNAs were extracted from $250 \mu 1$ serum using $750 \mu 1$ TRIzol $^{\circledR}$ LS reagent (Invitrogen; Thermo Fisher Scientific, Inc., Waltham, MA, USA) according to the manufacturer's protocol, and eluted with $35 \mu$ l pre-heated $\left(65^{\circ} \mathrm{C}\right)$ elution solution. A total of $10 \mu \mathrm{l}$ of Caenorhabditis elegans miR-39 $(0.05 \mu \mathrm{M})$ (synthesized by Shanghai GenePharma Co., Ltd., Shanghai, China) was added to each tube subsequent to serum mixing with TRIzol LS, and prior to the next step. Tissue RNA was isolated using TRIzol reagent according to the manufacturer's protocol, and eluted with $60 \mu 1$ pre-heated $\left(65^{\circ} \mathrm{C}\right)$ nuclease-free water. RNA quantification was carried out using NanoDrop 1000 (Thermo Fisher Scientific, Inc.).

Reverse transcription-quantitative polymerase chain reaction $(R T-q P C R)$. For miRNA, a Taqman MicroRNA Reverse Transcription kit (Applied Biosystems; Thermo Fisher Scientific, Inc.) was used to perform the reverse transcription reaction according to the manufacturer's protocol. qPCR reactions were performed in $20 \mu \mathrm{l}$ volume reaction containing $2 \mu \mathrm{l}$ cDNA, $10 \mu \mathrm{l}$ TaqMan 2X Perfect Master Mix (Takara Bio, Inc., Otsu, Japan), $0.5 \mu 1$ gene-specific primers/probe (Applied Biosystems; Thermo Fisher Scientific, Inc.) and $7.5 \mu 1$ nuclease-free water, and processed on a Bio-Rad IQ5 (Bio-Rad Laboratories, Inc., Hercules, CA, USA) thermocycler with the following parameters: $94^{\circ} \mathrm{C}$ for $1 \mathrm{~min}$, followed by 40 cycles of $94^{\circ} \mathrm{C}$ for $15 \mathrm{sec}$ and $60^{\circ} \mathrm{C}$ for $30 \mathrm{sec}$, and a melt curve with a range of 60 to $94^{\circ} \mathrm{C}$ and $0.5^{\circ} \mathrm{C}$ was raised in each analysis. For mRNA, PrimeScript RT reagent kits (Takara Bio, Inc.) and SYBR Green Realtime PCR Master Mix (Takara Bio, Inc.) were used according to the manufacturer's protocols. The $2^{-\Delta \Delta \mathrm{Cq}}$ method (18) was used to calculate the expression of miR-27a and SPRY2 relative to their references. The primer sequences were as follow: SPRY2-F: 5'-ATCCAGAGACAA GACATGTAC-3'; SPRY2-R: 5'-TTCAGATGTGTTCTA AGCC-3'; GAPDH-F: 5'-GCACCGTCAAGGCTGAGAAC-3'; GAPDH-R: 5'-GCCTTCTCCATGGTGGTGAA-3'. The primers of miR-27a and U6 were purchased from Shanghai GenePharma Co., Ltd.

Western blotting. LNCaP and PC-3 cells were lysed in radioimmunoprecipitation assay lysis buffer (Pierce; Thermo Fisher Scientific, Inc.) to extract total proteins which were measured using ABC kit (Thermo Fisher Scientific, Inc.) according to the manufacturer's protocols. A total of $60 \mu \mathrm{g}$ of total proteins were separated using SDS-PAGE (10\% gel) and transferred to polyvinylidene fluoride (PVDF) membranes. The PVDF membranes were blocked with $3 \%$ bovine serum albumin (Invitrogen; Thermo Fisher Scientific, Inc.) at room temperature for $1 \mathrm{~h}$ and then incubated with anti-SPRY2 (1:2,000; cat. no. ab50317, Upstate Biotechnology, Inc., Lake Placid, NY, USA) or GAPDH (1:1,000; cat. no. sc-293335, Santa Cruz Biotechnology, Inc., Dallas, TX, USA) antibodies at $4^{\circ} \mathrm{C}$ overnight. The PVDF membranes were then incubated with a horseradish peroxidase-conjugated secondary antibody (goat anti rabbit; cat no. ZB-2301; 1:10,000; Beijing Zhongshan Jinqiao Biotechnology Co., Ltd., Beijing, China) and finally detected using an enhanced chemiluminescence system (Amersham Pharmacia Biotech; GE Healthcare, Chicago, IL, USA) and ChemiDoc MP System with Image Lab Software version 6.0 (cat no. 170-8280; Bio-Rad Laboratories, Inc.).

Oligonucleotide transfection. The oligonucleotides were purchased from GenePharma, (Shanghai, China), including negative control, miR-27a inhibitor, miR-27a mimics, small interfering (si)-SPRY2 and SPRY2 vectors which contain the full-length SPRY2 cDNA sequence were constructed by Sangon Biotech (Shanghai, China). The sequences of oligonucleotides used were as follows: Negative control, 5'-UUCUCCGAACGUGUCACGUTT-3'; miR-27a inhibitors, 5'-GCGGAACUUAGCCACUGUGAA-3'; miR-27a mimics, 5'-UUCACAGUGGCUAAGUUCCGC-3'; si-SPRY2, 5'-CUC CAUUAGCUGAGUUCUAACAAG-3'. The oligonucleotides were transfected into LNCaP and PC-3 cells using Lipofectamine $^{\circledR} 2000$ (Invitrogen; Thermo Fisher Scientific, Inc.) according to the manufacturer's protocol. After $24 \mathrm{~h}$ of transfection, the LNCaP and PC-3 cells were collected to perform the experiments.

Cell proliferation assay. LNCaP and PC-3 cells were seeded at a density of 5,000/well in 96-well plates. The cells were transfected with the NC, miR-27a inhibitor, miR-27a mimics, si-SPRY2, or co-transfected with miR-27a mimics and a SPRY2 vector. Cell proliferation was analyzed following transfection for 1, 2, 3, 4 or 5 days, using Cell Counting Kit-8 (CCK8; Beyotime Institute of Biotechnology, Haimen, China) according to the manufacturer's protocol. 
A

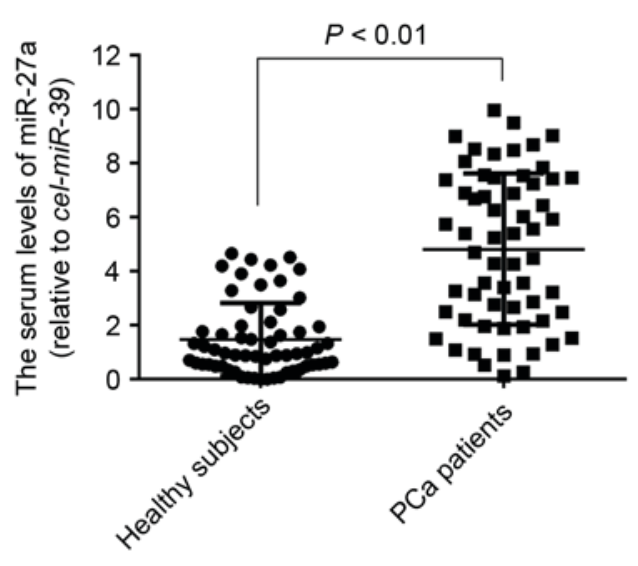

C

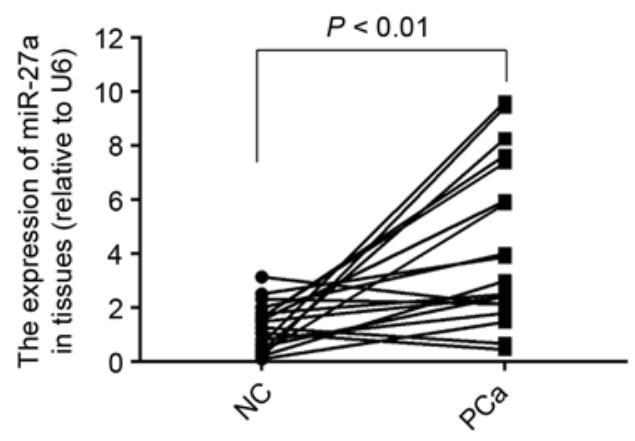

B

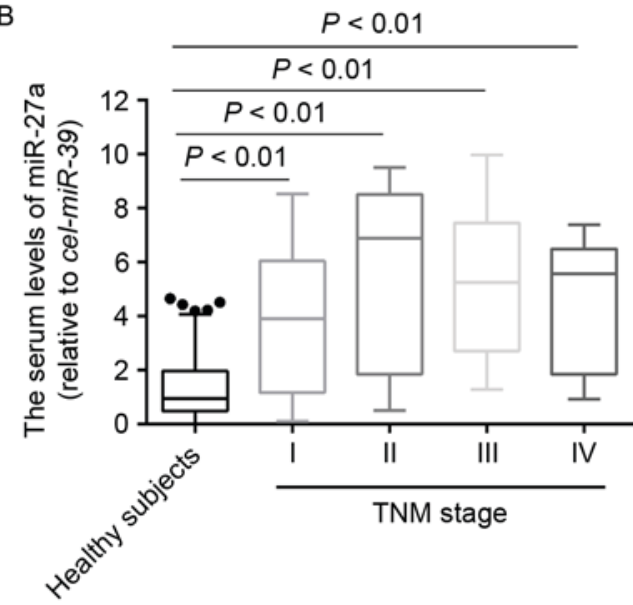

D

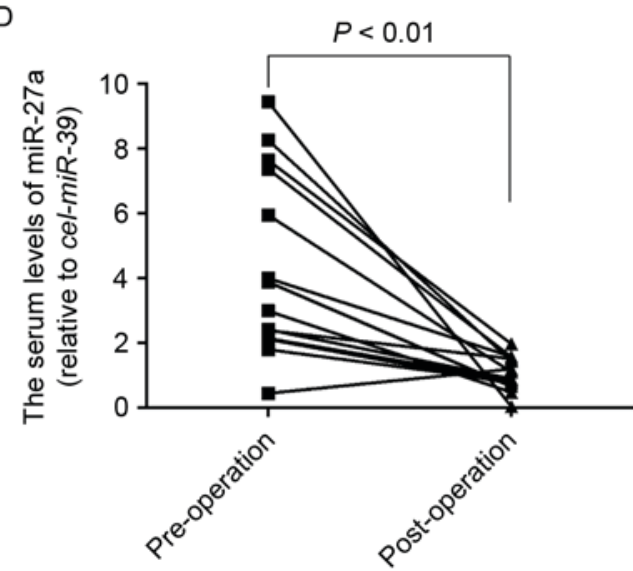

Figure 1. miR-27a is overexpressed in the sera and tumor tissue of patient with PCa. (A) The expression of miR-27a in the sera of 60 patients with PCa and 60 healthy subjects. (B) The expression of miR-27a in the sera of 60 healthy subjects and 60 PCa patients with different TNM stages, including stage I (12), stage II (13), stage III (25), stage IV (10). (C) The expression of miR-27a in 20 pairs of PCa tissues and matched normal tissues. (D) The expression of miR-27a in the sera of 14 patients with PCa prior or subsequent to operation for three months. PCa, prostate cancer; miR, microRNA; TNM, tumor node metastasis; $\mathrm{NC}$, negative control.

Cell cycle analysis. The cell cycle of PCa cells were analyzed using cell cycle and apoptosis analysis kit (Beyotime Institute of Biotechnology) according to the manufacturer's instruction. Briefly, $48 \mathrm{~h}$ after transfection with NC, miR-27a mimics or si-SPRY2, the cells were harvested and fixed in ice-cold 70\% ethanol overnight, and then the cells were resuspended in propidium staining solution (Beyotime Institute of Biotechnology) containing $40 \mu \mathrm{g} / \mathrm{ml}$ propidium iodide, $250 \mu \mathrm{g} / \mathrm{ml}$ RNase and $2 \mathrm{mM}$ EDTA, and incubated for $30 \mathrm{~min}$ at $37^{\circ} \mathrm{C}$. Cell cycle was analyzed using flow cytometer (FACSCalibur; BD Biosciences, Franklin Lakes, NJ, USA).

Statistical analysis.miR-27a expression in the serafrom patients and healthy subjects was compared using the Mann-Whitney test. miR-27a expression in pair tissues was analyzed using two-tailed Student's t-test. The one-way analysis of variance and the student-Newman-Keuls test was used to analyze more than two groups. Pearson's correlation was used to analyze the association between the expressions of miR-27a and SPRY2 mRNA. Receiver-operating characteristic (ROC) curves were used to assess the sensitivity/specificity of miR-27a for the diagnosis of $\mathrm{PCa} . \mathrm{P}<0.05$ was considered to indicate a statistically significant difference. All statistical analyses and graphs were performed and produced using GraphPad Prism 6.0 (GraphPad Software, Inc., La Jolla, CA, USA).

\section{Results}

miR-27a is overexpressed in the serum and tumor tissue of patients with $\mathrm{PCa}$. To the best of our knowledge, the expression of miR-27a in serum of patients with PCa remains unknown. To measure the serum levels of miR-27a, its expression was detected in 60 serum samples of patients with PCa and 60 healthy subjects. The results demonstrated that the serum levels of miR-27a were significantly higher in the patients with PCa compared with in the healthy subjects $(\mathrm{P}<0.01$; Fig. $1 \mathrm{~A})$, which was also observed in the PCa patients with stage I $(\mathrm{P}<0.01$; Fig. 1B), suggesting that the expression profiles of miR-27a in serum may act as novel non-invasive biomarkers for the diagnosis of early PCa. Furthermore, the expression of miR-27a in 20 pairs of PCa tissues and the matched normal tissues was also detected, and the results indicated that the expression of miR-27a was also significantly increased in the tumor tissues compared with the normal tissues (Fig. 1C). To determine whether miR-27a in serum was primarily derived from $\mathrm{PCa}$ tissues, the expression of miR-27a in 14 serum 


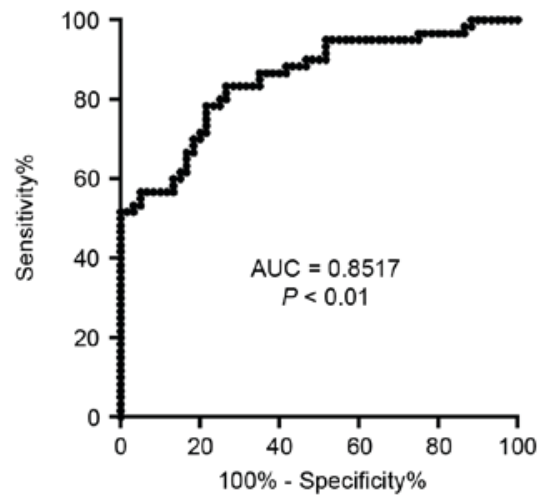

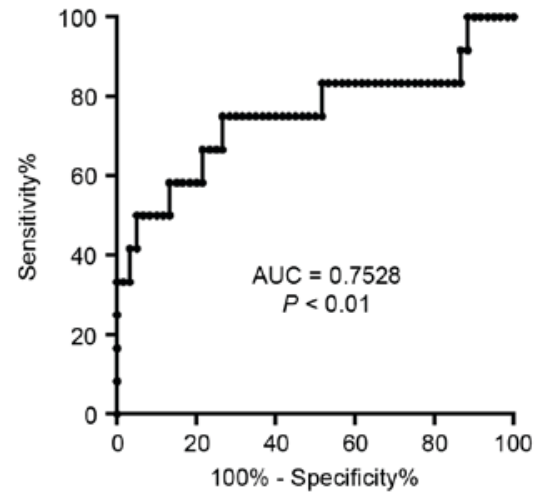

C

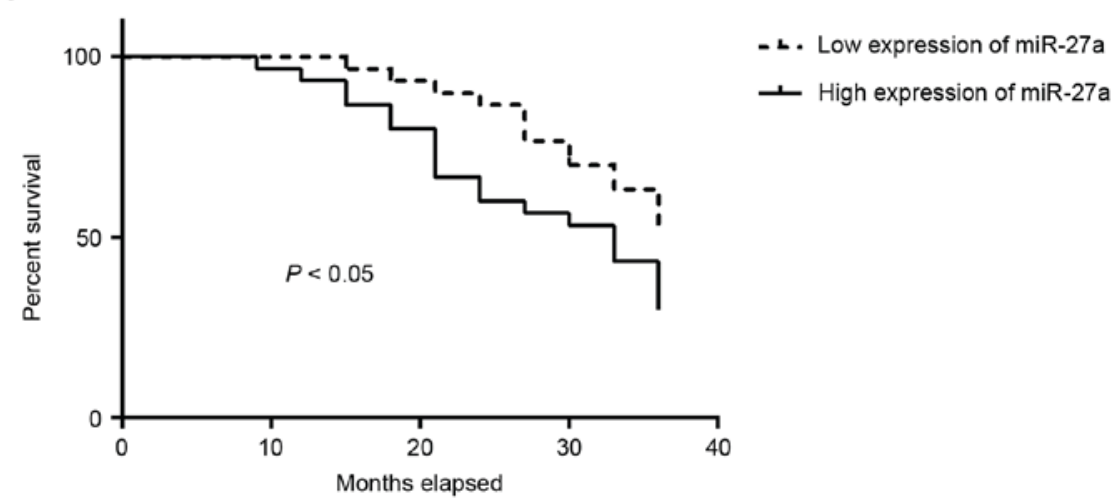

Figure 2. Serum level of miR-27a is a potential biomarker for the diagnosis of early PCa and is associated with poor survival of patients. (A) Receiver operating characteristic curve analysis reveals that the serum level of miR-27a in distinguishing 60 healthy subjects from 60 patients with PCa demonstrated an AUC of 0.8517, and (B) in distinguishing 60 healthy subjects from 12 patients with stage I PCa demonstrated an AUC of 0.7528. (C) Survival rate analysis reveals that patients with PCa with high expression of miR-27a have poor survival rates. AUC, areas under the curve; PCa, prostate cancer; miR, microRNA.

A

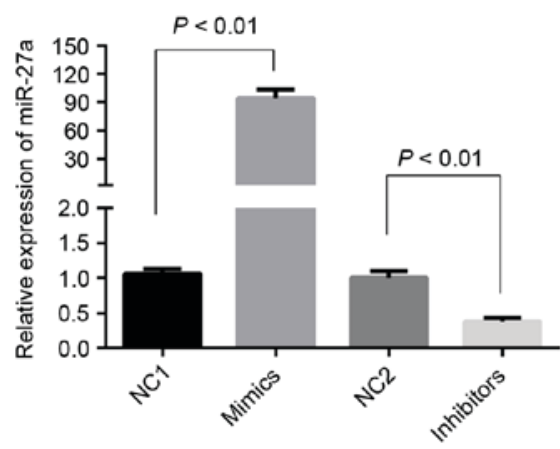

C

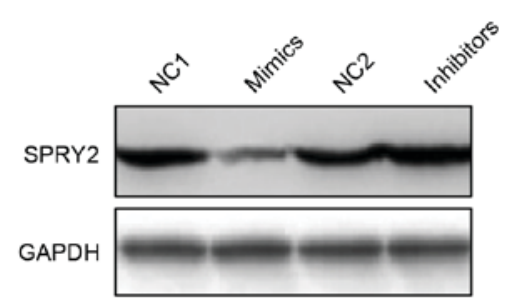

B
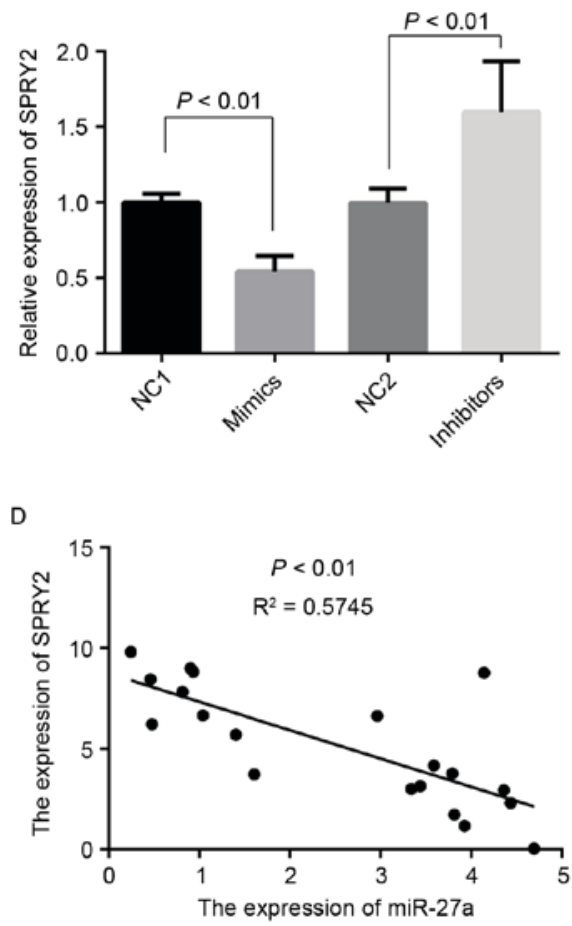

Figure 3. miR-27a directly inhibits SPRY2 expression, and the expression levels of these two proteins are inversely correlated in PCa tissues. The mRNA expressions of (A) miR-27a or (B) SPRY2 in PCa cells transfected with NC1 (negative control for mimics), Mimics (miR-27a mimics), NC2 (negative control for inhibitors) or inhibitors (miR-27a inhibitors), respectively. (C) The protein expression of SPRY2 in PCa cells. (D) The correlation between the mRNA expressions of miR-27a and SPRY2 in 20 PCa tissues. miR, microRNA; SPRY2, Sprouty2; PCa, prostate cancer; NC, negative control. 


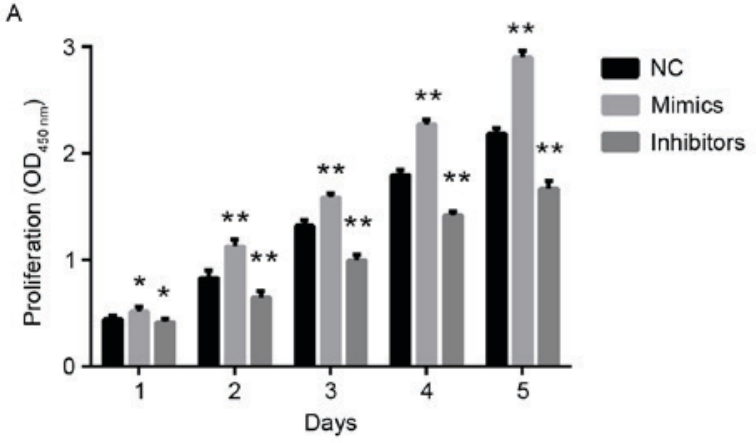

C

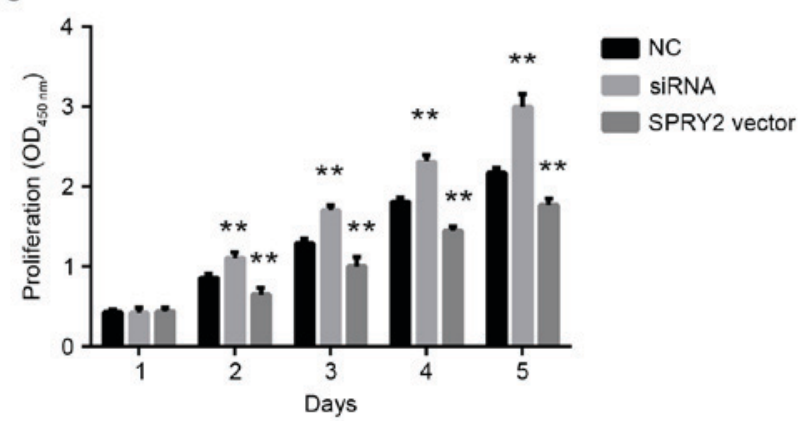

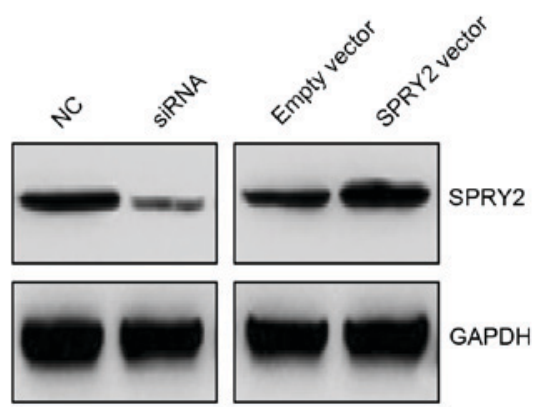

D

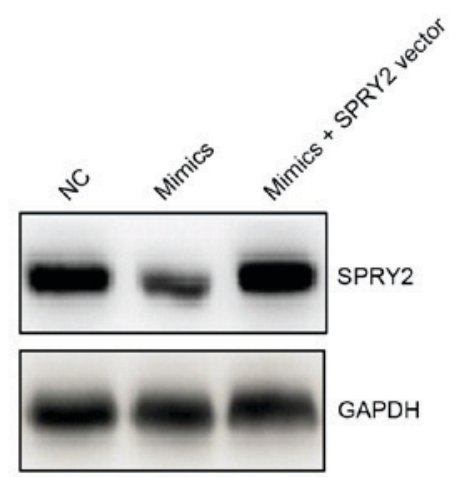

$\mathrm{E}$

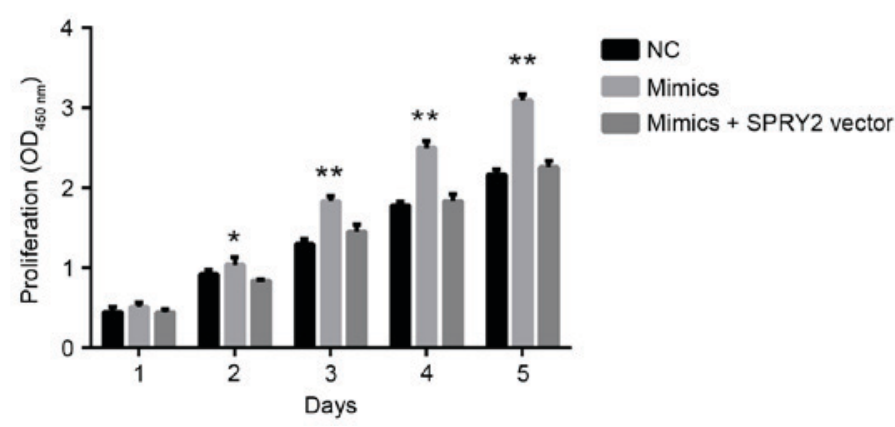

Figure 4. miR-27a promotes the proliferation activity of PCa cells and this function is rescued by the overexpression of SPRY2. (A) The proliferation of PCa cells transfected with NC, miR-27a mimics or miR-27a inhibitors by 1 to 5 days, respectively. ${ }^{*} \mathrm{P}<0.05$ vs. NC; ** $\mathrm{P}<0.01$ vs. NC. (B) The protein expression of SPRY2 in PCa cells transfected with NC, SPRY2 siRNA, Empty vector or SPRY2 vector for 48 h, respectively. (C) The proliferation of PCa cells transfected with NC, SPRY2 siRNA or SPRY2 vector for 1 to 5 days, respectively. (D) The protein expression of SPRY2 in PCa cells transfected with NC, miR-27a mimics or miR-27a mimics + SPRY2 vector by $48 \mathrm{~h}$, respectively. ${ }^{* *} \mathrm{P}<0.01$ vs. NC. (E) The proliferation of PCa cells transfected with NC, miR-27a or miR-27a mimics + SPRY2 for 1 to 5 days, respectively. ${ }^{*} \mathrm{P}<0.05$ vs. NC; ${ }^{* *} \mathrm{P}<0.01$ vs. NC. miR, microRNA; SPRY2, Sprouty2; PCA, prostate cancer; si, small interfering; NC, negative control.

samples of patients who underwent surgery was measured for 3 months, and the results demonstrated that the serum levels of miR-27a was significantly decreased compared with the pre-operative levels (Fig. 1D), suggesting that the increased levels of miR-27a in serum was caused by the PCa tissues.

Serum level of miR-27a is a potential biomarker for the diagnosis of early PCa and associated with poor survival of patients. The diagnostic capabilities of the expression profiles of miR-27a in the sera in distinguishing patients with PCa from normal subjects were additionally analyzed. ROC curve analyses revealed that the serum level of miR-27a was a valuable biomarker for distinguishing patients with $\mathrm{PCa}$ from healthy subjects, with AUC of 0.8517 [95\% confidence interval (CI): 0.7842-0.9191]. The cutoff value of 2.136 was considered optimal, and the sensitivity and specificity were 78.33 and $78.33 \%$, respectively (Fig. 2A). The serum level of miR-27a was also a valuable biomarker for distinguishing PCa patients with TNM stage I from the healthy subjects with AUC of 0.7528 (95\% CI: 0.5681-0.9375). The cutoff value of 1.854 was considered optimal, and the sensitivity and specificity were 75 and $73.33 \%$, respectively (Fig. 2B). It was then determined whether the serum level of miR-27a was associated with patient survival. The 60 patients with PCa were divided into two groups, low expression of miR-27a (range, 0.1109-4.6972) and high expression of miR-27a (range, 5.2489-9.9653), using the median expression of miR-27a. The analysis of patient survival between the two groups was performed. The results indicated that patients with PCa with high expression of miR-27a exhibited significantly poorer survival rates compared with those with low expression $(\mathrm{P}<0.05$; Fig. 2C). These data suggested that the expression profile of miR-27a in serum may be a potential biomarker for the diagnosis and prognosis of patients with $\mathrm{PCa}$. 

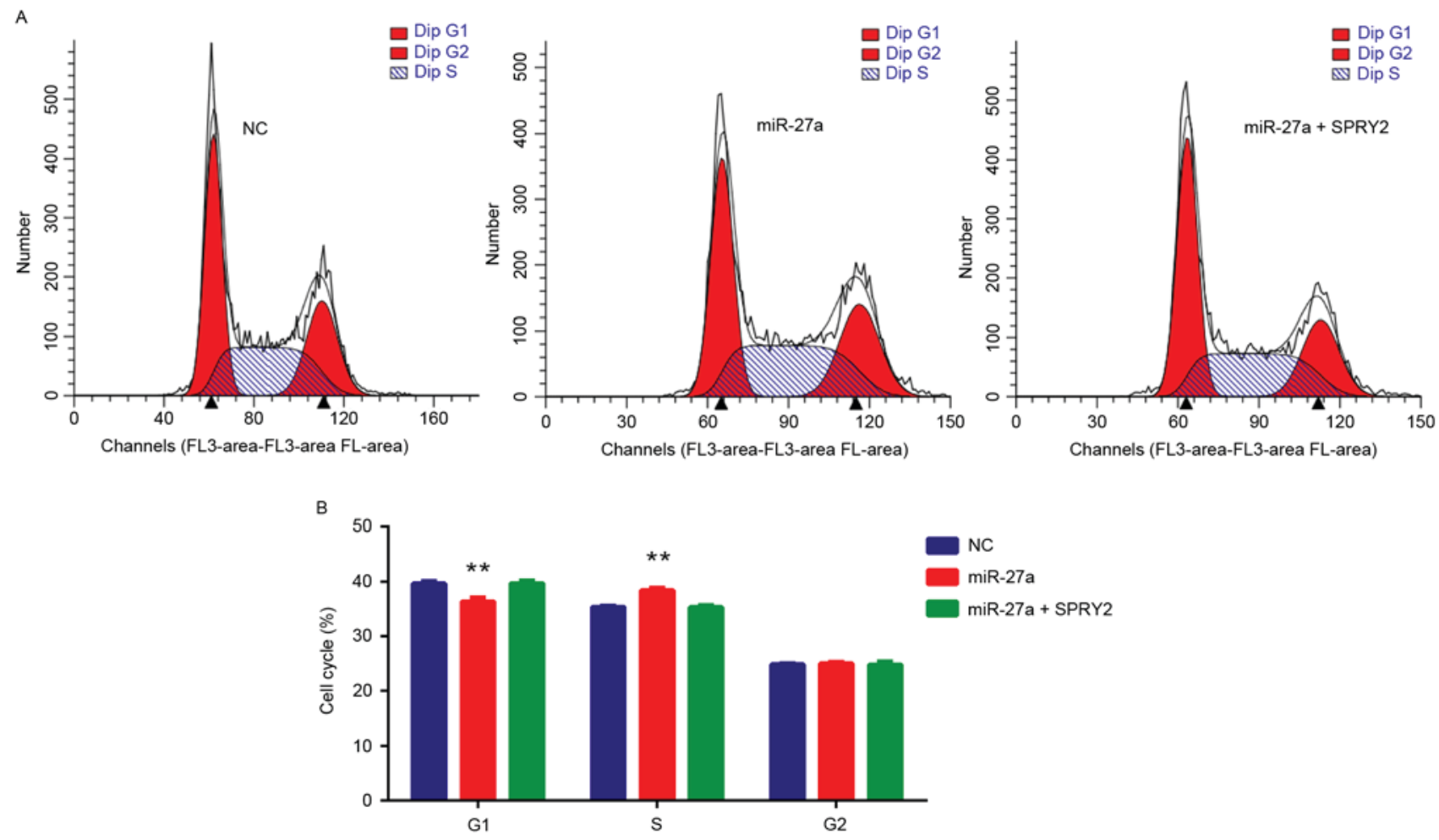

Figure 5. miR-27a promotes the cell cycle of PCa cells. (A) Flow cytometry reveals the cell cycle of PCa cells, including G1, S and G2 stages. (B) The PCa cells transfected with miR-27a mimics exhibited a higher number of S-stage cells compared with those transfected with the NC, and the overexpression of SPRY2 rescued miR-27a-induced high S stage. ${ }^{* *} \mathrm{P}<0.01$ vs. NC. miR, microRNA; SPRY2, Sprouty2; PCa, prostate cancer; NC, negative control.

miR-27a directly inhibits SPRY2 expression, and their levels of expression are inversely correlated in PCa tissues. SPRY2, a tumor suppressor gene, is downregulated in PCa tissues, and SPRY2 overexpression may suppress cell proliferation in prostate cancer (19). Additionally, SPRY2 is hypothesized to be a target of miR-27a: miR-27a altered cell growth, colony formation and migration in pancreatic cancer through directly targeting SPRY2 (12). However, it is unclear whether the expression of SPRY2 is regulated by miR-27a in PCa cells. Therefore, the expression of SPRY2 was initially detected in the PCa cells transfected with miR-27a mimics or inhibitors (Fig. 3A). Compared with the negative control groups, the levels of mRNA and protein expression of SPRY2 were markedly decreased, and increased, respectively (Fig. 3B and C). The correlation between the expression levels of miR-27a and SPRY2 were additionally analyzed in PCa tissues, and the results demonstrated that the expression of miR-27a was negatively associated with SPRY2 (Fig. 3D). These data suggested that miR-27a/SPRY2 axis may serve an essential role in the proliferation of PCa cells.

miR-27a promotes the proliferation activity of PCa cells and this function is rescued by the overexpression of SPRY2. miR-27a was suggested to act as an oncomiR and affect PCa cell growth (11); therefore the present study analyzed the function of miR-27a in PCa cells, particularly the miR-27a/SPRY2 axis. The miR-27a mimics or inhibitors were transfected into PCa cells, and the results revealed that miR-27a mimics significantly promoted the proliferation of PCa cells, whereas miR-27a inhibitors significantly suppressed the proliferation of PCa cells compared with the negative control groups (Fig. 4A), suggesting that miR-27a did act as an oncomiR in PCa. The expression of SPRY2 was also inhibited or overexpressed by using specific siRNA or SPRY2-overexpressed vectors in PCa cells, respectively (Fig. 4B), and the results indicated that the downregulation of SPRY2 significantly increased the proliferation of PCa cells, whereas the upregulation of SPRY2 significantly decreased the proliferation of PCa cells (Fig. 4C). To additionally investigate whether miR-27a exerting its function depended on the expression of SPRY2, miR-27a mimics and SPRY2-overexpressed vectors were co-transfected into PCa cells (Fig. 4D), and the results demonstrated that the proliferation of PCa cells was rescued compared with the $\mathrm{PCa}$ cells transfected with miR-27a mimics only (Fig. 4E). These data suggested that the miR-27a/SPRY2 axis served an important role in the proliferation of PCa cells.

miR-27a/SPRY2 axis regulates the cell cycle of PCa cells. Whether miR-27a/SPRY2 did affect the cell cycle of PCa cells was additionally investigated, and the results revealed that miR-27a mimics significantly increased the number of S-stage cells $(\mathrm{P}<0.01)$, whereas when the cells were co-transfected with miR-27a mimics and SPRY2 vectors, the number of S-stage cells was rescued compared with the negative control (Fig. 5). These data suggested that the miR-27a/SPRY2 axis serves an important role in the cell cycle of PCa cells.

\section{Discussion}

The present study identified that the expression of miR-27a was increased in the tumor tissue and serum of patients with $\mathrm{PCa}$, and was correlated with poor survival of patients with 
PCa. Furthermore, it was determined that miR-27a acted as an oncomiR and promoted the proliferation activity of $\mathrm{PCa}$ cells. In addition, miR-27a directly inhibited the mRNA and protein expression of SPRY2, which is considered as a tumor suppressor in PCa. In the majority of different types of cancer, miR-27a serves as an oncomiR and promoted the progression of cancers. Fletcher et al (11) first identified that androgen regulated the overexpression of miR-27a in $\mathrm{PCa}$ and that its upregulation was involved in cell growth. However, Walter et al (10) demonstrated that the downregulation of miR-27 was present in high-grade tumors. This contradictory phenomenon formed the basis of the present study, to investigate the in vivo expression of miR-27a in tumor tissue of patients with PCa. The data indicated that miR-27a was overexpressed in PCa tissues. In addition, the data demonstrated that the overexpression of miR-27a was also present in the sera of patients with PCa compared to the healthy subjects. Furthermore, the data revealed that the overexpression of miR-27a in serum was associated with poor survival of patients with PCa. These suggested that the expression profile of miR-27a in sera may be a potential biomarker for the diagnosis and prognosis of patients with $\mathrm{PCa}$.

SPRY was identified in Drosophila as an inhibitor of fibroblast growth factor signaling (20). SPRY2 was first identified to be epigenetically suppressive in prostate cancer and to act as a tumor suppressor (21). Although Ma et al (12) suggested that SPRY2 is a direct target of miR-27a in pancreatic cancer, the association between SPRY2 and miR-27a in PCa remains unclear. The data of the present study indicated that the expression of miR-27a was inversely correlated with the expression of SPRY2 in PCa tissues, and miR-27a promoted the proliferation of $\mathrm{PCa}$ cells also through inhibiting the expression of SPRY2, suggesting that the miR-27a/SPRY2 axis serves an important role in the proliferation of PCa cells. It was also hypothesized that SPRY2 is a direct target of miR-27a. In addition, Liu et al (22) demonstrated that SPRY2 is also a direct target of miR-27b, a relative of miR-27a. Due to this, SPRY2 was not identified a direct target of miR-27a in the present study.

miRNAs serum profiles are valuable biomarkers for the diagnosis of diseases, due to its non-invasion, stability and specificity. The challenge is that there is no suitable internal reference to normalize the expression of miRNAs in sera, but using exogenous genes as reference may be feasible, including Cel-miR-39, a miRNA in nematodes $(17,23,24)$. The present study measured the relative expression of miR-27a in the sera of patients with PCa and healthy subjects using this approach. The data indicated that the serum levels of miR-27a were significantly correlated with the survival rate of patients with PCa. In the present study, a limitation was that the number of samples was small. Although 140 subjects were enrolled, including 80 patients with PCa and 60 healthy subjects, the conclusion that the expression profile of miR-27a in serum acted as novel non-invasive biomarker for the diagnosis of $\mathrm{PCa}$ needs to be confirmed in large scale in the future.

Taken together, the overexpression of miR-27a was identified in the tumor tissue and serum of patients with $\mathrm{PCa}$ and was correlated with poor survival of patients. Furthermore, miR-27a as an oncomiR promoted the proliferation and cell cycle of PCa cells by targeting SPRY2. These suggested that the serum signature of miR-27a may be a novel non-invasive biomarker for the diagnosis of PCa and the miR-27a/SPRY2 axis may be a therapeutic target.

\section{Acknowledgements}

Not applicable.

\section{Funding}

The present study was supported by grants from the Project of Jiangxi Provincial Education Department (grant nos. YC2012-S026 and GJJ14077).

\section{Availability of data and materials}

The datasets generated and analyzed in the present study are included in this published article.

\section{Authors' contributions}

WG and $\mathrm{ZH}$ performed the experiments. $\mathrm{HH}, \mathrm{AZ}$, and SL collected the patient samples. CC and XZ analyzed the data. GZ and ZS designed the study and wrote this article.

\section{Ethics and consent to participate}

All specimens were collected from the individuals who provided written informed consent according to the protocols approved by the Ethics Review Board at Nanchang University (Nanchang, China).

\section{Consent for publication}

The study participants provided consent for the data to be published.

\section{Competing interests}

The authors declare that they have no competing interests.

\section{Author information}

No additional information provided.

\section{References}

1. DeSantis C, Naishadham D and Jemal A: Cancer statistics for African Americans, 2013. CA Cancer J Clin 63: 151-166, 2013.

2. Hayes JH and Barry MJ: Screening for prostate cancer with the prostate-specific antigen test: A review of current evidence. JAMA 311: 1143-1149, 2014.

3. Siegel RL, Miller KD and Jemal A: Cancer statistics, 2016. CA Cancer J Clin 66: 7-30, 2016.

4. Lin XJ, Chong Y, Guo ZW, Xie C, Yang XJ, Zhang Q, Li SP, Xiong Y, Yuan Y, Min J, et al: A serum microRNA classifier for early detection of hepatocellular carcinoma: A multicentre, retrospective, longitudinal biomarker identification study with a nested case-control study. Lancet Oncol 16: 804-815, 2015.

5. Li BS, Zuo QF, Zhao YL, Xiao B, Zhuang Y, Mao XH, Wu C, Yang SM, Zeng H, Zou QM and Guo G: MicroRNA-25 promotes gastric cancer migration, invasion and proliferation by directly targeting transducer of ERBB2, 1 and correlates with poor survival. Oncogene 34: 2556-2565, 2015. 
6. Sapre N, Macintyre G, Clarkson M, Naeem H, Cmero M, Kowalczyk A, Anderson PD, Costello AJ, Corcoran NM and Hovens CM: A urinary microRNA signature can predict the presence of bladder urothelial carcinoma in patients undergoing surveillance. Br J Cancer 114: 454-462, 2016.

7. Farazi TA, Hoell JI, Morozov P and Tuschl T: MicroRNAs in human cancer. Adv Exp Med Biol 774: 1-20, 2013.

8. Sharova E, Grassi A, Marcer A, Ruggero K, Pinto F, Bassi P, Zanovello P,Zattoni F, D'Agostino DM, Iafrate $\mathrm{M}$ and Ciminale $\mathrm{V}$ : A circulating miRNA assay as a first-line test for prostate cancer screening. Br J Cancer 114: 1362-1366, 2016.

9. Yin C, Fang C, Weng H, Yuan C and Wang F: Circulating microRNAs as novel biomarkers in the diagnosis of prostate cancer: A systematic review and meta-analysis. Int Urol Nephrol 48: 1087-1095, 2016

10. Walter BA, Valera VA, Pinto PA and Merino MJ: Comprehensive microRNA profiling of prostate cancer. J Cancer 4: 350-357, 2013.

11. Fletcher CE, Dart DA, Sita-Lumsden A, Cheng H, Rennie PS and Bevan CL: Androgen-regulated processing of the oncomir miR-27a, which targets prohibitin in prostate cancer. Hum Mol Genet 21: 3112-3127, 2012.

12. Ma Y, Yu S, Zhao W, Lu Z and Chen J: miR-27a regulates the growth, colony formation and migration of pancreatic cancer cells by targeting Sprouty2. Cancer Lett 298: 150-158, 2010.

13. Peng H, Wang X, Zhang P, Sun T, Ren X and Xia Z: miR-27a promotes cell proliferation and metastasis in renal cell carcinoma. Int J Clin Exp Pathol 8: 2259-2266, 2015.

14. Salah Z, Arafeh R, Maximov V, Galasso M, Khawaled S, Abou-Sharieha S, Volinia S, Jones KB, Croce CM and Aqeilan RI: miR-27a and miR-27a* contribute to metastatic properties of osteosarcoma cells. Oncotarget 6: 4920-4935, 2015.

15. Zhao N, Sun H, Sun B, Zhu D, Zhao X, Wang Y, Gu Q, Dong X, Liu F, Zhang Y and Li X: miR-27a-3p suppresses tumor metastasis and VM by down-regulating VE-cadherin expression and inhibiting EMT: An essential role for Twist-1 in HCC. Sci Rep 6: 23091, 2016.
16. Bostwick DG: Staging prostate cancer-1997: Current methods and limitations. Eur Urol 32 (Suppl 3): S2-S14, 1997.

17. Li BS, Zhao YL, Guo G, Li W, Zhu ED, Luo X, Mao XH, Zou QM, Yu PW, Zuo QF, et al: Plasma microRNAs, miR-223, miR-21 and miR-218, as novel potential biomarkers for gastric cancer detection. PLoS One 7: e41629, 2012.

18. de Barros Pita W, Leite FC, de Souza Liberal AT, Pereira LF, Carazzolle MF, Pereira GA and de Morais MA Jr: A new set of reference genes for RT-qPCR assays in the yeast Dekkera bruxellensis. Can J Microbiol 58: 1362-1367, 2012.

19. Patel R, Gao M, Ahmad I, Fleming J, Singh LB, Rai TS, McKie AB, Seywright M, Barnetson RJ, Edwards J, et al: Sprouty2, PTEN, and PP2A interact to regulate prostate cancer progression. J Clin Invest 123: 1157-1175, 2013.

20. Hacohen N, Kramer S, Sutherland D, Hiromi Y and Krasnow MA: sprouty encodes a novel antagonist of FGF signaling that patterns apical branching of the Drosophila airways. Cell 92: 253-263, 1998.

21. McKie AB, Douglas DA, Olijslagers S, Graham J, Omar MM, Heer R, Gnanapragasam VJ, Robson CN and Leung HY: Epigenetic inactivation of the human sprouty2 (hSPRY2) homologue in prostate cancer. Oncogene 24: 2166-2174, 2005

22. Liu C, Liang S, Xiao S, Lin Q, Chen X, Wu Y and Fu J: MicroRNA-27b inhibits Spry2 expression and promotes cell invasion in glioma U251 cells. Oncol Lett 9: 1393-1397, 2015.

23. Mitchell PS, Parkin RK, Kroh EM, Fritz BR, Wyman SK, Pogosova-Agadjanyan EL, Peterson A, Noteboom J, O'Briant KC, Allen A, et al: Circulating microRNAs as stable blood-based markers for cancer detection. Proc Natl Acad Sci USA 105: 10513-10518, 2008.

24. Xiao B, Wang Y, Li W, Baker M, Guo J, Corbet K, Tsalik EL, Li QJ, Palmer SM, Woods CW, et al: Plasma microRNA signature as a non-invasive biomarker for acute graft-versus-host disease. Blood 122: 3365-3375, 2013. 